\title{
Significant Traumatic Intracranial Hemorrhage in the Setting of Massive Bee Venom-Induced Coagulopathy: A Case Report
}

\author{
Kelsey Stack, DO; Lindsey Pryor, MD \\ From the Department of Emergency Medicine, SUNY Upstate Medical University, Syracuse, NY.
}

\begin{abstract}
Bees and wasps of the Hymenoptera order are encountered on a daily basis throughout the world. Some encounters prove harmless, while others can have significant morbidity and mortality. Hymenoptera venom is thought to contain an enzyme that can cleave phospholipids and cause significant coagulation abnormalities. This toxin and others can lead to reactions ranging from local inflammation to anaphylaxis. We report a single case of a previously healthy man who presented to the emergency department with altered mental status and anaphylaxis after a massive honeybee envenomation that caused a fall from standing resulting in significant head injury. He was found to have significant coagulopathy and subdural bleeding that progressed to near brain herniation requiring emergent decompression. Trauma can easily occur to individuals escaping swarms of hymenoptera. Closer attention must be paid to potential bleeding sources in these patients and in patients with massive bee envenomation.
\end{abstract}

Key words: hymenoptera, coagulopathy, anaphylaxis, traumatic subdural hemorrhage

\section{Introduction}

High venom loads, known as "massive envenomations," result from $>50$ stings to a victim during a single encounter. This can produce what is known as a toxic reaction, a potentially life-threatening complication that induces anything from rhabdomyolysis to disseminated intravascular coagulation. We present a case of a patient who experienced significant intracranial bleeding and complications immediately following massive bee envenomation, which produced coagulopathy after head trauma.

\section{Case Presentation}

A 71-year-old man with no prior medical history for significant disease presented to the emergency department (ED) with altered mental status, a reported fall, and anaphylaxis. The patient was a groundskeeper at a local

Corresponding author: Lindsey Pryor, MD, SUNY Upstate University Hospital, Department of Emergency Medicine, Suite 103, 550 East Genesee Street, Syracuse NY 13202 (e-mail: pryorl@upstate.edu).

Submitted for publication January 2016

Accepted for publication May 2016 high school where he was using a weed-whacker in a level grass field when he disturbed a beehive. His coworkers reported they found him on the ground where he had either tripped and fallen from standing or syncopized; he was covered in honeybees to the extent that bystanders were unable to see his face or neck. The school nurse administered intramuscular epinephrine $0.3 \mathrm{mg}$ prior to emergency medical services (EMS) arrival as he appeared unresponsive to bystanders.

Upon EMS arrival, the patient was combative, with altered mental status, and found to be in acute anaphylaxis with reported vital sign abnormalities including a blood pressure of $96 / 70 \mathrm{~mm} \mathrm{Hg}$, heart rate of 120 beats/min, and respiratory rate of 20 breaths $/ \mathrm{min}$ with wheezing. His initial Glasgow Coma Scale was 10 (eye-4, verbal-1, motor-6) but improved within 5 minutes to a GCS of 14 (eye-4, verbal-4, motor-6). These findings prompted EMS to give a second dose of intramuscular $0.3 \mathrm{mg}$ epinephrine, intravenous (IV) methylprednisolone $125 \mathrm{mg}$, and $1 \mathrm{~L}$ normal saline bolus in the field. Diphenhydramine was not given in the prehospital setting.

In the ED, the patient was agitated, pulling at his cervical collar, and disoriented to place and time. Initial 
vital signs were blood pressure $131 / 75 \mathrm{~mm} \mathrm{Hg}$, heart rate 98 beats/min, respiration rate 25 breaths/min, and oxygen saturation $96 \%$ on facemask. On physical examination, a laceration was noted to the back of his head with controlled bleeding and scalp bogginess with equal but sluggishly reactive pupils. Faint wheezes were auscultated throughout all lung fields. He was initially treated with diphenhydramine $50 \mathrm{mg} \mathrm{IV}$, ranitidine 50 mg IV, albuterol nebulizer, and $1 \mathrm{~L}$ normal saline fluids for the allergic reaction, and was administered lorazepam $1 \mathrm{mg}$ IV for agitation.

A computed tomography (CT) scan of the head was done within 1 hour of arrival and revealed a right occipital skull facture with associated small left frontal subdural hemorrhage (see Figure 1). Coagulation studies were abnormal with partial thromboplastin time (PTT) $>180$ seconds, prothrombin time (PT) 21.3 seconds, and international normalized ratio (INR) of 1.7. The initial etiology of these altered bleeding times was unclear at the time as the patient had no known bleeding disorders and took no anticoagulants per the patient's spouse, who was present to give the patient's medical history. A discussion ensued between the emergency medicine physicians and neurosurgical team regarding a possible laboratory abnormality or an altered blood draw while the patient was initially being stabilized, since normal saline was running through the IV simultaneously. The PT, PTT, and INR were all reordered, but due to high patient volume and numerous intensive care unit holds in the ED, these diagnostic studies were delayed and not sent in a timely manner. Hemoglobin and

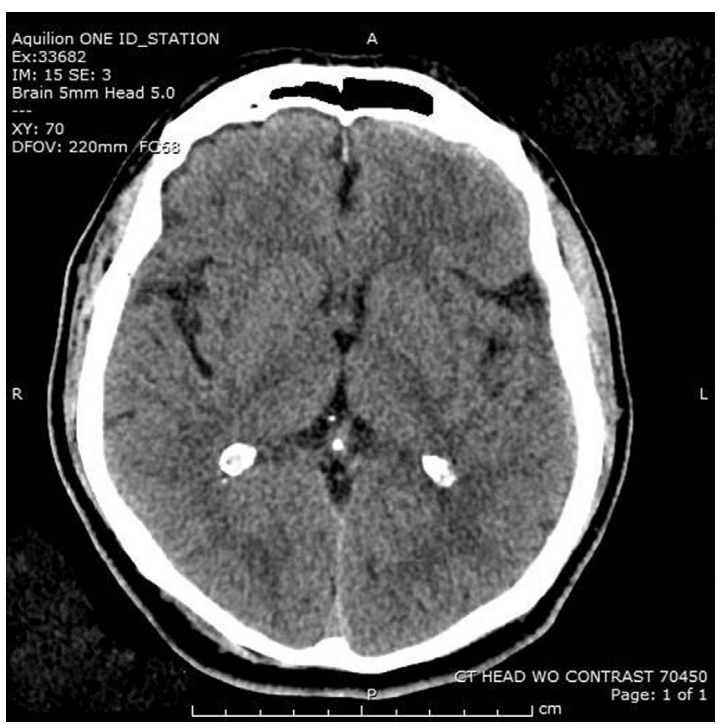

Figure 1. The initial computed tomography scan of our patient, done within the first hour after bee attack, showing a small left frontal subdural hemorrhage. hematocrit were $13.6 \mathrm{~g} / \mathrm{dL}$ and $40.2 \%$, respectively, and platelet count was $234 \times 10^{3} / \mu \mathrm{L}$. The patient had no signs of renal impairment with a blood urea nitrogen of $17 \mathrm{mg} / \mathrm{dL}$ and creatinine of $1.0 \mathrm{mg} / \mathrm{dL}$. Given the small intracranial hemorrhage no operative management was indicated and no coagulation reversal was advised despite acknowledgment of abnormal laboratory results. Supportive care and a repeat CT of the head in 6 hours were recommended. At that time, the patient became much more cooperative and alert and was answering questions more appropriately.

Due to lack of intensive care unit bed availability, the patient remained in the ED 5 hours after arrival. He was continually reassessed, and the nursing staff noted a decline in mental status at the 5.5 hour mark that included moaning and making nonpurposeful movements. The decision was made to repeat a CT scan emergently. Before the scan, he had equal pupillary response. Repeat imaging showed significant increase in the subdural hemorrhage with midline shift and brain compression (see Figure 2). Rapid sequence intubation was performed using etomidate $20 \mathrm{mg}$ and succinylcholine $100 \mathrm{mg}$ with successful intubation, and prothrombin complex concentrate (KCentra, CSL Behring) 2500 units IV and mannitol $25 \% 100 \mathrm{~g}$ was administered in the ED. The hematology service was consulted for the coagulation abnormalities. They surmised that this patient's altered bleeding times were secondary to his significant amount of bee venom exposure. They also postulated these abnormal bleeding times contributed to his traumatic subdural hemorrhage. When the repeat coagulation values did result, they

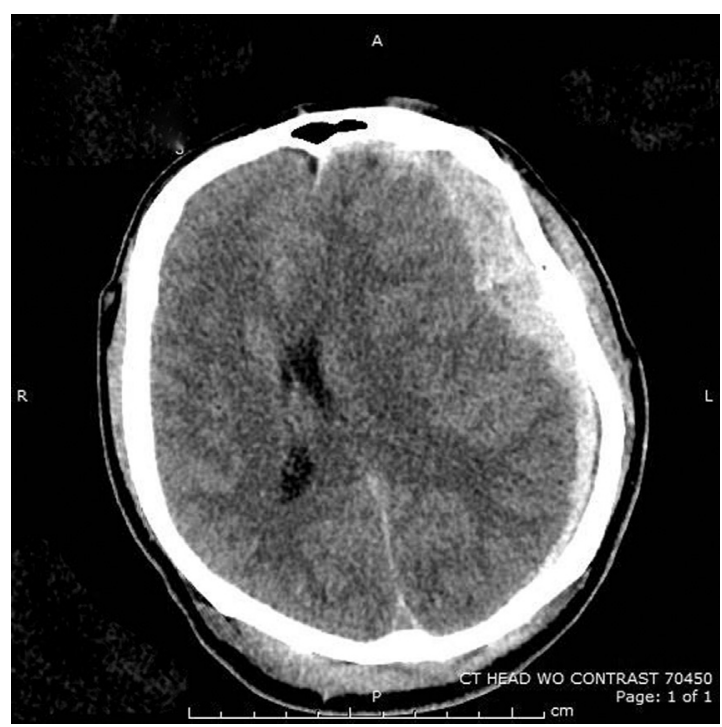

Figure 2. The patient's second computed tomography scan, done about 5.5 hours later, showing significant increase in subdural hemorrhage with midline shift and mass effect. 
remained abnormal with PT 20.8 seconds, INR 1.7 seconds, and PTT 42.9 seconds. The patient was taken to the operating room where he was additionally given 4 units of fresh frozen plasma and underwent emergent decompressive craniotomy and evacuation of hematoma 6.5 hours after initial evaluation.

\section{Discussion}

The Hymenoptera order includes Apoidea (over 20,000 species of bees) and Vespoidea (which includes wasps and hornets). The main difference between these groups is that Apoidea, or honeybees, are able to sting only once because of their barbed stinger and are much less aggressive than the wasp or hornet group. Colony size is also varied, and disruption of a wasp colony may result in tens to hundreds of stings versus the several thousand stings that are possible with honeybee colony disruption, especially with Africanized honeybees. ${ }^{1}$

While most practicioners are well versed in treating anaphylatic reactions, this case highlights a rare complication secondary to massive envenomation from honeybees. Only 2 deaths related to envenomations from bees, wasps, and hornets were reported to the National Data Poisions System from 2008-2011. This low number is thought to be attributed to improved awareness and recognition of allergic reactions and better access to medical care. ${ }^{2}$ Massive envenomations can occur in nonallergic individuals with the lethal dose being 500 to $1200+$ stings per adult ${ }^{1}$ or 20 stings $/ \mathrm{kg}$ in mammals ${ }^{3}$; however, death has been reported to occur in as few as 250 stings from Africanized bees. ${ }^{4}$

There are generally 4 types of reactions to bee venom from a single sting. The local reaction is a nonimmunoglobulin E (IgE)-related reaction that causes local edema and swelling with symptoms usually resolving within 24 hours. A regional reaction is caused by mast cell degranulation, causing extensive erythema and swelling of an entire limb, for example-a reaction that may take up to 24 hours to develop. A systemic reaction manifests as anaphylaxis and includes urticaria, angioedema, hypotension, vomiting, and shortness of breath, all occurring within 10 to 15 minutes of the stings. ${ }^{1,5}$ For individuals with immediate hypersensitivity reactions, a single sting can cause death from an anaphylactic reaction in individuals who have produced IgE antibodies to the venom from a previous sting, a reaction which is not dose-dependent. ${ }^{6}$ The fourth type of reaction is serum sickness, which generally occurs 3 days to 2 weeks after envenomations and is related to cirulating immune complexes and manifests as vasculitis, arthritis, neuropathy, Guillian-Barre syndrome, thrombotic thrombocytopenic purpura, and disseminated intravascular coagulation. ${ }^{6-9}$

Massive envenomations can cause a toxic reaction, which is not IgE-mediated but rather is secondary to direct effects of the unusually high dose of venom injected into the victim, as in the case of our patient. This manifests with symptoms of gastrointestinal upset, headache, syncope, rhabdomyolysis, acute kidney injury, and seizures, while bronchospasm and uticaria are less common. These systemic effects include hemolysis and acute tubular necrosis, which lead to elevated creatine phosphokinase and rhabdomyolysis. Death from systemic toxic effects can occur anywhere from 16 hours to 12 days after bee attack and largely results from renal failure secondary to extensive hemolysis and myolysis. $^{1,10}$

Bee venom is made up of protein, enzymes, and amines. One of the major components, mellitin, is a protein that hydrolyzes cell membranes and causes histamine release. This protein is thought to be responsible for local pain reaction and also causes a catecholamine release. ${ }^{6}$ Phospholipase $\mathrm{A}_{2}\left(\mathrm{PLA}_{2}\right)$, the other major component, is believed to inhibit cellular functions by hydrolyzing 2-acyl bonds in natural lipids, including in phospholipids of mitochondria and other cell constituents. ${ }^{4}$ The effects of this polypeptide chain causes contraction of smooth muscles, lowering of blood pressure, capillary permeability, and destruction of mast cells and is likely the main culprit in coagulation abnormalities. ${ }^{2,4}$ Hider $^{11}$ postulates that PLA 2 inactivates phospholipids that normally help blood coagulation factors to bind together and clot blood, a disruption that results in impaired hemostasis. Petroianu et $\mathrm{al}^{4}$ found a correlation between high concentrations of $\mathrm{PLA}_{2}$ in human plasma and prolonged PT, activated PTT, and antithrombin III, a finding reflected in our case in which PTT was initially found to be $>180$ seconds.

The mainstay of treatment remains supportive care, airway management, and treatment of anaphylaxis with epinephrine, diphenhydramine, and steroids. Removal of stingers has no impact on venom delivery to the victim since venom typically empties from detached bee stingers within 10 to 20 seconds; however, retained stingers may have potential for foreign body-induced infection in the nonacute setting. ${ }^{1,12}$

The risk of toxic envenomations appears to be related to the number of bee stings a patient experiences and should be considered when taking a history and ordering laboratory tests. For the more serious toxic reactions from massive envenomations, special consideration needs to be made with regard to potential for kidney injury, rhabdomyolysis, and coagulation derangements. If trauma is suspected, as was apparent in our case, serial coagulation 
studies may guide the need for further treatment and potential anticoagulation reversal using fresh frozen plasma, cryoprecipitate, prothrombin complex concentrate, or any of the newer recombinant concentrates.

Hospital observation is recommended for individuals in the extremes of age or with multiple comorbidities presenting with $>100$ stings. $^{13}$ Some poison control centers recommend that older patients, pediatric patients, and patients with multiple co-morbidities presenting with $>50$ stings should be admitted for 24-hour observation as they have an increased risk of delayed tissue injury. Otherwise healthy patients with 50 or more stings who are only experiencing pain should be observed in the ED with diagnostic studies performed at initial presentation and at 6 hours after to rule out hemolysis, thrombocytopenia, kidney injury, and elevated creatine kinase. ${ }^{14}$

Our case is unique among other reports of massive envenomation since the patient experienced head trauma during the initial attack, with a lucid period in the ED, and then rapidly decompensated. Although it is reasonable to conclude that subdural hemorrhage expansion is expected in the setting of trauma alone, we believe this patient's complication was exacerbated by the adverse effects of the large dose of bee venom on his clotting ability. Review of the medical literature found 1 case report in which a patient presented with a nontraumatic cerebellar, subarachnoid, and parenchymal hemorrhage 2 days after being stung by 8 to 10 bees; however, coagulation studies were normal. ${ }^{15}$ In contrast, our case illustrates a scenario of a minor traumatic fall from standing, along with coagulation abnormalities immediately after innumerable bee stings and seriously exacerbated brain hemorrhage within 6 hours of ED arrival. This demonstrates the importance of considering coagulation defects as a major complication due to a toxic reaction from numerous honeybee stings.

Acknowledgments: We would like to acknowledge general support by Emergency Department at SUNY Upstate staff member, Gary Johnson, MD, FACEP; the associate professor and chair of Emergency Medicine; as well as review and editing support from William Paolo, $\mathrm{MD}$, the residency program director and associate professor; and Susan Wojcik, PhD, ATC. Thank you to Jennifer Kruse, DO, for her contributions to this case.

Author Contributions: Study concept and design (KS); acquisition of the data (LP, KS); analysis of the data (LP); drafting of the manuscript
(LP); critical revision of the manuscript (KS); and approval of final manuscript (KS).

Financial/Material Support: None.

Disclosures: None.

\section{References}

1. Vetter RS, Visscher PK, Camazine S. Mass envenomations by honey bees and wasps. West J Med. 1999;170: 223-227.

2. Hahn I. Arthropods. In: Hoffman RS, Howland MA, Lewin NA, Nelson LS, Goldfrank LR, eds. Goldfrank's Toxicologic Emergencies. 10th ed. New York, NY: McGraw-Hill; 2015.

3. Manoquerra AS. Hymenoptera stings. In: Ling L, Clark RF, Erickson TB, Trestrail JH, eds. Toxicology Secrets. Philadelphia, PA: Hanley and Belfus; 2001.

4. Petroianu G, Liu J, Helfrich U, Maleck W, Rüfer R. Phospholipase A2-induced coagulation abnormalities after bee sting. Am J Emerg Med. 2000;18:22-27.

5. Rathoff OD, Nossel HL. Wasp sting anaphylaxis. Blood. 1983;61:132-139.

6. Fitzgerald KT, Flood AA. Hymenoptera stings. Clin Tech Small Anim Pract. 2006;21:194-204.

7. Gawlik R, Rymarczyk B, Rogala B. A rare cause of intravascular coagulation after honey bee sting. J Invest Allergol Clin Immunol. 2004;14:250-252.

8. Ashley JR, Otero H, Aboulafia DM. Bee envenomation: a rare cause of thrombotic thrombocytopenic purpura. South Med J. 2003;96:588-591.

9. Jung JW, Jeon EJ, Kim JW, et al. A fatal case of intravascular coagulation after bee sting acupuncture. Allergy Asthma Immunol Res. 2012;4:107-109.

10. Rosenfeld G. Human envenomation by bee stings. In: Rosenberg P, ed. Toxins: Animal, Plant and Microbial. Oxford, UK: Pergamon Press; 1976:692-693.

11. Hider RC. Honeybee venom: a rich source of pharmacologically active peptides. Endeavour. 1988;12:60-65.

12. Schumacher MJ, Tveten MS, Egen NB. Rate and quantity of delivery of venom from honeybee stings. J Allergy Clin Immunol. 1994;93:831-835.

13. Schneir AB. Bites and stings. In: Tintinalli JE, Stapczynski JS, Ma OJ, Yealy DM, Meckler GD, Cline DM, eds. Tintinalli's Emergency Medicine: A Comprehensive Study Guide. New York, NY: McGraw-Hill; 2011.

14. Kolecki P. Delayed toxic reaction following massive bee envenomation. Ann Emerg Med. 1999;33:114-116.

15. Yoder J, Lloyd A, Tilney PV, McKenna DP. Intracerebral hemorrhage following multiple bee stings. JAAPA. 2013;26. 